\title{
Energy spectra of carbon and oxygen cosmic rays with CALET on the International Space Station
}

Paolo Maestro ${ }^{a, b, *}$ on behalf of the CALET Collaboration

(a complete list of authors can be found at the end of the proceedings)

${ }^{a}$ Department of Physical Sciences, Earth and Environment, University of Siena, via Roma 56, 53100 Siena, Italy

${ }^{b}$ INFN Sezione di Pisa, Polo Fibonacci, Largo B. Pontecorvo 3, 56127 Pisa, Italy

E-mail: maestro@unisi.it

We present the measurements of the energy spectra of carbon and oxygen nuclei in cosmic rays based on 4 years of observation with the Calorimetric Electron Telescope (CALET) on the International Space Station. The energy spectra are measured from $10 \mathrm{GeV} / n$ to $2.2 \mathrm{TeV} / n$ with an all calorimetric instrument with a total thickness corresponding to 1.3 nuclear interaction length and equipped with charge detectors capable of single element resolution. Data analysis, including the detailed assessment of systematic uncertainties, and results are reported. The observed carbon and oxygen fluxes show a spectral hardening around $200 \mathrm{GeV} / n$ established with a significance $>3 \sigma$. They have the same energy dependence and a constant $\mathrm{C} / \mathrm{O}$ flux ratio above $25 \mathrm{GeV} / \mathrm{n}$. These measurements will contribute to a better understanding of the origin of the spectral hardening.

$37^{\text {th }}$ International Cosmic Ray Conference (ICRC 2021)

July 12 th - 23rd, 2021

Online - Berlin, Germany

\footnotetext{
${ }^{*}$ Presenter
} 


\section{Introduction}

The CALorimetric Electron Telescope (CALET) [1] is a space-based instrument installed on the International Space Station from August 2015. The instrument is optimized for the measurement of the all-electron spectrum [2,3], but it can also measure the flux of individual chemical elements in cosmic rays (CR) from proton to iron in the energy range up to $\sim 1 \mathrm{PeV}$, searching for possible spectral hardening as already observed in the proton spectrum [4].

In this paper, we present a new direct measurement of the CR carbon and oxygen spectra from 10 $\mathrm{GeV} / n$ to $2.2 \mathrm{TeV} / n$, based on the data collected from October 13, 2015 to October 31, 2019.

\section{Detector}

CALET instrument comprises a CHarge Detector (CHD), a finely segmented pre-shower IMaging Calorimeter (IMC), and a Total AbSorption Calorimeter (TASC). A complete description of the instrument can be found in [2].

The IMC consists of 7 tungsten plates inserted between eight double layers of $1 \mathrm{~mm}^{2}$ crosssection scintillating fibers, arranged in belts along orthogonal directions. Fiber signals are used to reconstruct the $\mathrm{CR}$ particle trajectory and its entrance point in the instrument, by means of a track finding and fitting algorithm based on a combinatorial Kalman filter [5]. The angular resolution is $\sim 0.1^{\circ}$ for $\mathrm{C}$ and $\mathrm{O}$ nuclei and the spatial resolution on the determination of the impact point on CHD is $\sim 220 \mu \mathrm{m}$.

The identification of the particle charge $Z$ is based on the measurements of the ionization deposits in CHD and IMC. CHD is comprised of two hodoscopes made of 14 plastic scintillator paddles each, arranged in orthogonal layers (CHDX, CHDY). The particle trajectory is used to identify the CHD paddles and IMC fibers traversed by the primary particle and to determine the path length correction to be applied to the signals to extract the $d E / d x$ samples. Three charge values $\left(Z_{\mathrm{CHDX}}\right.$, $\left.Z_{\mathrm{CHDY}}, Z_{\mathrm{IMC}}\right)$ are reconstructed, on an event-by-event basis, from the measured $d E / d x$ in each CHD layer and the average of $d E / d x$ samples (at most eight) along the track in the top half of IMC [6]. The charge resolution $\sigma_{Z}$ is $\sim 0.15 e$ (charge unit) for CHD and $\sim 0.24 e$ for IMC, respectively, in the elemental range from $\mathrm{B}$ to $\mathrm{O}$.

The TASC is a homogeneous calorimeter made of 12 layers of lead-tungstate bars readout by photosensors and a front-end electronics spanning a dynamic range $>10^{6}$. The total thickness of the instrument is equivalent to 30 radiation length and 1.3 nuclear interaction length. The TASC response was studied at CERN SPS in 2015 using a beam of accelerated ion fragments with $A / Z=2$ and kinetic energy of 13,19 and $150 \mathrm{GeV} / n$ [7]. The response curve for triggered particles of each nuclear species is nearly gaussian at a fixed beam energy. The mean energy released in the TASC is $\sim 20 \%$ of the particle energy and the resolution is $\sim 30 \%$. The energy response of TASC turned out to be linear up to the maximum particle energy $(6 \mathrm{TeV})$ available at the beam, as described in [8]. Monte Carlo (MC) simulations, reproducing the detailed detector configuration, physics processes, as well as detector signals, are based on the EPICS simulation package [9] and employ the hadronic interaction model DPMJET-III [10]. Independent simulations based on FLUKA [11] and Geant4 10.5 [12] are used to assess the systematic uncertainties. The energy response derived from MC simulations was tuned using the beam test results. 


\section{Data analysis}

We have analyzed flight data (FD) collected by the high-energy (HE) shower trigger of the instrument in the first 1480 days of CALET operation aboard the ISS. The HE trigger is based on the coincidence of the summed signals of the last two IMC layers in each view and the top TASC layer (TASCX1), with thresholds corresponding to 50 and 100 times the signal released by a minimum ionizing particle (MIP) in IMC and TASC, respectively.

Events with one well-fitted track crossing the whole detector from CHD top to the TASC bottom layer and at least $2 \mathrm{~cm}$ away from the edges are then selected. The fiducial geometrical factor for this category of events is $S \Omega \sim 510 \mathrm{~cm}^{2} \mathrm{sr}$, corresponding to about $50 \%$ of the total CALET acceptance. Particle entering the detector from lateral sides or late-interacting in the bottom half of TASC are rejected by analyzing the longitudinal and lateral shower profiles.

Carbon and oxygen candidates are selected by applying window cuts, centered on the nominal charge values $(Z=6,8)$, of half-width $0.4 e$ for $Z_{C H D X}$ and $Z_{C H D Y}$, and $2 \sigma_{Z}$ for $Z_{I M C}$, respectively. Particles undergoing a charge-changing nuclear interaction in the upper part of the instrument are removed by the three combined charge selections and by requiring that the average of $d E / d x$ measurements in the first four layers in each IMC view are consistent within $30 \%$.

$6.154 \times 10^{5} \mathrm{C}$ and $1.047 \times 10^{6} \mathrm{O}$ candidate events are identified by this selection procedure. For flux measurement, energy unfolding is applied to correct the distributions of energy deposit in TASC $\left(E_{\mathrm{TASC}}\right.$ ) by selected $\mathrm{C}$ and $\mathrm{O}$ nuclei for significant bin-to-bin migration effects (due to the limited energy resolution) and infer the primary particle energy. The energy spectrum is obtained from the unfolded energy distribution as follows:

$$
\begin{gathered}
\Phi(E)=\frac{N(E)}{\Delta E \varepsilon(E) S \Omega T} \\
N(E)=U\left[N_{o b s}\left(E_{\mathrm{TASC}}\right)-N_{b g}\left(E_{\mathrm{TASC}}\right)\right]
\end{gathered}
$$

where: $\Delta E$ is the energy bin width; $E$ the particle kinetic energy, calculated as the geometric mean of the lower and upper bounds of the bin; $N(E)$ the bin content in the unfolded distribution; $T$ the exposure time (84.5\% of total observation time); $\varepsilon(E)$ the total selection efficiency; $U()$ the iterative unfolding procedure based on Bayes' theorem [13]; $N_{o b s}\left(E_{\mathrm{TASC}}\right)$ the bin content of observed energy distribution (including background); $N_{b g}\left(E_{\mathrm{TASC}}\right)$ the bin content of background events in the observed energy distribution. Background contamination from different nuclear species misidentified as $\mathrm{C}$ or $\mathrm{O}$ is $<0.1 \%$ in all energy bins with $E_{\mathrm{TASC}}<10^{3} \mathrm{GeV}$, and between $0.1 \%$ and $1 \%$ for $E_{T A S C}>10^{3} \mathrm{GeV}$.

\section{Systematic Uncertainties}

In this analysis, dominant sources of systematic uncertainties include trigger efficiency, energy response, event selection, unfolding procedure, MC model.

The HE trigger efficiency as a function of $E_{\text {TASC }}$ was measured using a subset of data taken with the Low-Energy gamma (LEg) trigger which has the same HE trigger logic but lower thresholds (5 and 10 MIP for IMC and TASC, respectively), allowing to trigger also penetrating particles. $\mathrm{HE}$ efficiency curves for $\mathrm{C}$ and $\mathrm{O}$ are consistent with predictions from $\mathrm{MC}$ simulations [8]. In 
order to study the flux stability against offline trigger efficiency, the threshold applied to TASCX1 signal was scanned between 100 and 150 MIP signal. The corresponding systematic errors range between $-4.2 \%(-3.1 \%)$ and $3.7 \%(7.3 \%)$ for $\mathrm{C}(\mathrm{O})$ depending on the energy bin. Moreover the $\mathrm{C}$ and $\mathrm{O}$ fluxes were also measured using datasets taken with different trigger modes: the LEg and the LEe (a dedicated trigger for electrons operated at high geomagnetic latitudes) with livetimes corresponding to $10 \%$ and $2 \%$ of the HE trigger livetime, respectively. The resultant fluxes from the different trigger modes analyses show consistent normalization and spectral shapes.

The systematic error related to charge identification was studied by varying the width of the window cuts between $0.35 e$ and $0.45 e$ for CHD and between $1.75 \sigma_{Z}$ and $2.2 \sigma_{Z}$ for IMC. That results in a flux variation depending on the energy bin, which is less than $1 \%$ below $250 \mathrm{GeV} / n$ and few percent above. The ratio of events selected by IMC charge cut to the ones selected with CHD in different $E_{\text {TASC }}$ intervals turned out to be consistent in FD and MC.

The uncertainty in the energy scale is $\pm 2 \%$ and depends on the accuracy of the beam test calibration. It causes a rigid shift of the measured energies, affecting the absolute normalization of the $\mathrm{C}$ and O spectra by ${ }_{-2.8 \%}^{+2.6 \%}$, but not their shape. As the beam test model was not identical to the instrument now in orbit, the difference in the spectrum obtained with either configuration was modeled and included in the systematic error.

Other energy-independent systematic uncertainties affecting the normalization include live time (3.4\%) and long-term stability of the charge measurements $(<0.4 \%)$.

The uncertainties due to the unfolding procedure were evaluated by using different response matrices computed by varying the spectral index (between -2.9 and -2.5) of the generation spectrum of MC simulations.

Since it is not possible to validate MC simulations with beam test data in the high-energy region, a comparison between different MC models, i.e. EPICS and FLUKA, was performed. We found that the total selection efficiencies for $\mathrm{C}$ and $\mathrm{O}$ determined with the two models are in agreement within $<1.5 \%$ over the whole energy range, but the energy response matrices differ significantly in the low and high energy regions. The resulting fluxes show maximum discrepancies of $9 \%(7.8 \%)$ and $9.2 \%(12.2 \%)$, respectively, in the first and last energy bin for $\mathrm{C}(\mathrm{O})$, while they are consistent within $6.6 \%(6.2 \%)$ elsewhere. This is the dominant source of systematic uncertainties. We also check that energy spectra obtained from different analyses using EPICS, FLUKA and Geant4 simulations are consistent in terms of absolute normalization and spectral shape.

Materials traversed by nuclei in IMC are mainly composed of carbon, aluminum and tungsten. Possible uncertainties in the inelastic cross sections in simulations or discrepancies in the material description might affect the flux normalization. We have checked that hadronic interactions are well simulated in the detector, by measuring the survival probabilities of $\mathrm{C}$ and $\mathrm{O}$ nuclei at different depths in IMC, as described in [8]. The survival probabilities are in agreement with MC prediction within $<1 \%$.

The total systematic error is computed as the sum in quadrature of all the sources of systematics in each energy bin. 


\section{Results}

The energy spectra of carbon and oxygen and their flux ratio measured with CALET in an energy range from $10 \mathrm{GeV} / n$ to $2.2 \mathrm{TeV} / n$ are shown in Fig. 1. Our spectra are consistent with PAMELA [17] and most previous experiments [15, 16, 20-22]. CALET and AMS-02 C and O spectra differ in the absolute normalization, which is lower for CALET by about $27 \%$ for both $\mathrm{C}$ and $\mathrm{O}$. However they have very similar shapes as indicated by the very consistent measurements of the $\mathrm{C} / \mathrm{O}$ flux ratio.

Figure 2 shows the fits to CALET carbon and oxygen data with a double power-law function

$$
\Phi(E)= \begin{cases}C\left(\frac{E}{\mathrm{GeV}}\right)^{\gamma} & E \leq E_{0} \\ C\left(\frac{E}{\mathrm{GeV}}\right)^{\gamma}\left(\frac{E}{E_{0}}\right)^{\Delta \gamma} & E>E_{0}\end{cases}
$$

where $C$ is a normalization factor, $\gamma$ the spectral index, and $\Delta \gamma$ the spectral index change above the transition energy $E_{0}$. A single power-law (SPL) function (Eq.3 with $\Delta \gamma=0$ ) fitted to data in the energy range $[25,200] \mathrm{GeV} / n$ and extrapolated above $200 \mathrm{GeV} / n$ is also shown for comparison. The effect of systematic uncertainties in the measurement of the energy spectrum is modeled in the $\chi^{2}$ minimization function with a set of 6 nuisance parameters [8]. The DPL fit to the C spectrum yields a spectral index $\gamma=-2.663 \pm 0.014$ at energies below the transition region $E_{0}=(215 \pm 54)$ $\mathrm{GeV} / n$ and a spectral index increase $\Delta \gamma=0.166 \pm 0.042$ above, with $\chi^{2} /$ d.o.f. $=9.0 / 8$. For oxygen, the fit yields $\gamma=-2.637 \pm 0.009, E_{0}=(264 \pm 53) \mathrm{GeV} / n, \Delta \gamma=0.158 \pm 0.053$, with $\chi^{2} /$ d.o.f. $=$ 3.0/8. SPL fits give $\gamma=-2.626 \pm 0.010$ with $\chi^{2} /$ d.o.f. $=27.5 / 10$ for $\mathrm{C}$, and $\gamma=-2.622 \pm 0.008$ with $\chi^{2} /$ d.o.f. $=15.9 / 10$ for $\mathrm{O}$, respectively. A frequentist test statistic $\Delta \chi^{2}$ is computed from the difference in $\chi^{2}$ between the fits with SPL and DPL functions. For carbon (oxygen), $\Delta \chi^{2}=18.5$ (12.9) with 2 d.o.f. (i.e. the number of additional free parameters in DPL fit with respect to SPL fit) implies that the significance of the hardening of the $\mathrm{C}(\mathrm{O})$ spectrum exceeds the $3 \sigma$ level.

In order to study the energy dependence of the spectral index in a model independent way, the spectral index $\gamma$ is calculated by a fit of $d[\log (\Phi)] / d[\log (E)]$ in energy windows centered in each bin and including the neighbor \pm 3 bins. The results in Fig. 3 show that carbon and oxygen fluxes harden in a similar way above a few hundred $\mathrm{GeV} / n$. The carbon to oxygen flux ratio is well fitted to a constant value of $0.911 \pm 0.006$ above $25 \mathrm{GeV} / n$, indicating that the two fluxes have the same energy dependence [8].

\section{Conclusion}

With a calorimetric apparatus in low Earth orbit, CALET has measured the energy spectra of carbon and oxygen nuclei in CR and their flux ratio from $10 \mathrm{GeV} / n$ to $2.2 \mathrm{TeV} / n$. Our observations show a spectral index increase $\Delta \gamma=0.166 \pm 0.042(0.158 \pm 0.053)$ in the $\mathrm{C}(\mathrm{O})$ spectrum above 200 $\mathrm{GeV} / n$, allowing to exclude a single power law spectrum by more than $3 \sigma$. C and $\mathrm{O}$ fluxes have the same energy dependence and a constant $\mathrm{C} / \mathrm{O}$ flux ratio $0.911 \pm 0.006$ above $25 \mathrm{GeV} / n$. Our results are consistent with the ones reported by AMS-02. However the absolute normalization of our data is significantly lower than AMS-02, but in agreement with other experiments. We performed detailed systematic checks to search for possible causes of this normalization issue. We can exclude that 


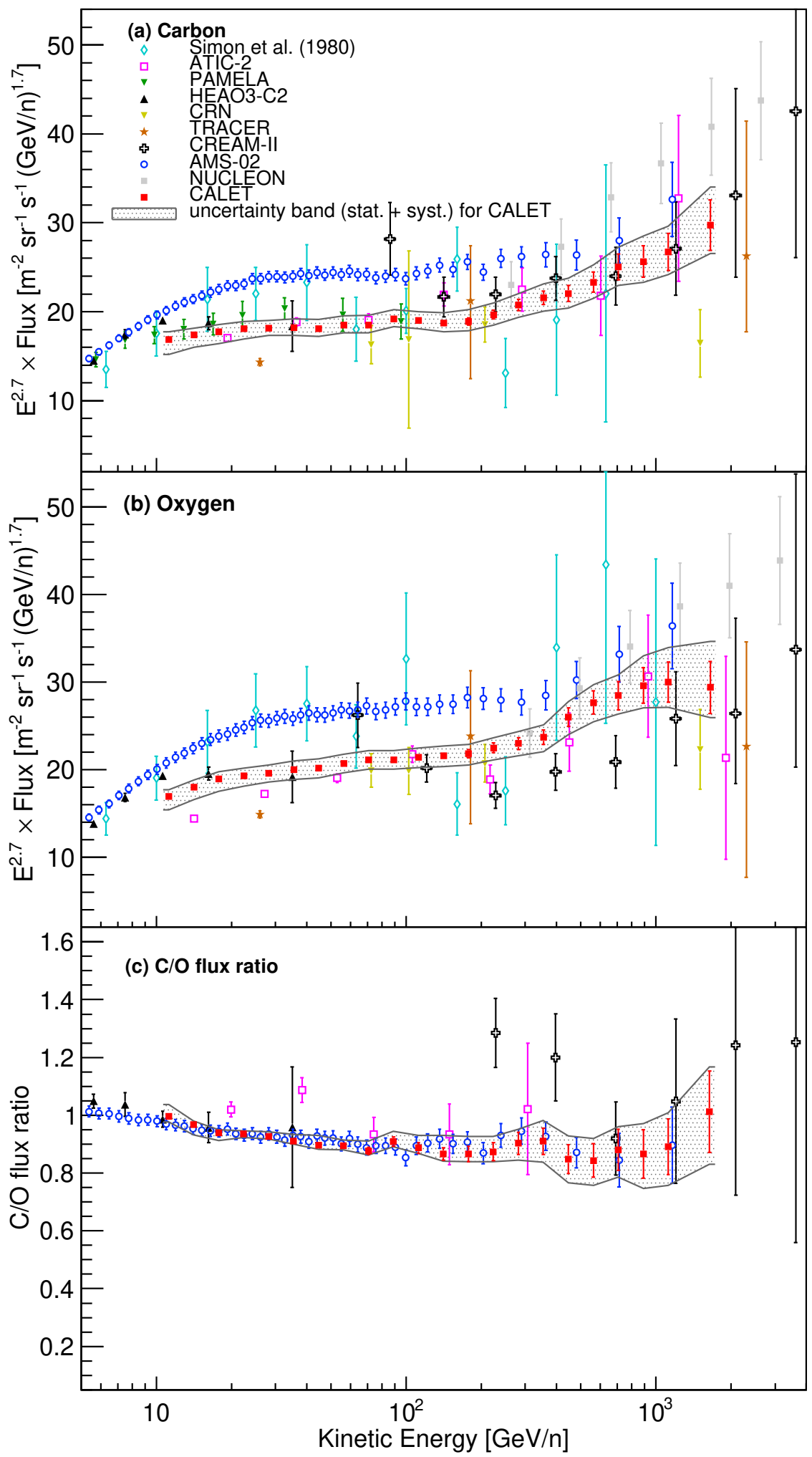

Figure 1: CALET (a) carbon and (b) oxygen flux (multiplied by $E^{2.7}$ ) and (c) ratio of carbon to oxygen fluxes, as a function of kinetic energy $E$. Error bars of CALET data (red) represent the statistical uncertainty only, while the gray band indicates the quadratic sum of statistical and systematic errors. Also plotted are other direct measurements [14-22]. 


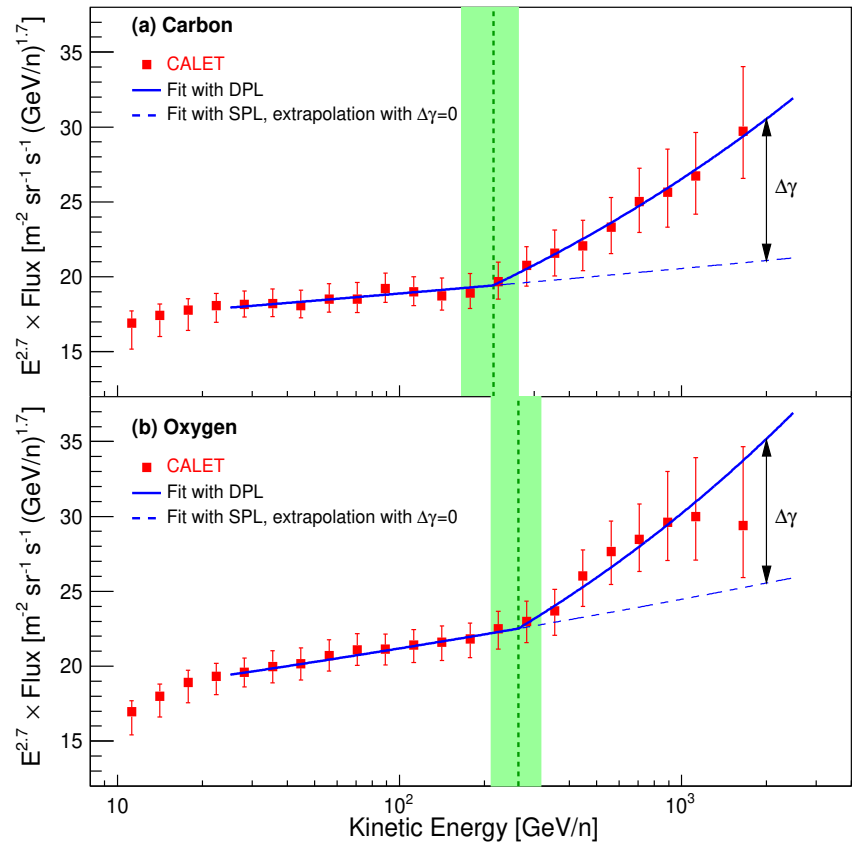

Figure 2: Fit of the CALET (a) C and (b) O energy spectra with a DPL function (Eq.3, blue line) in the energy range [25, 2000] $\mathrm{GeV} / n$. The flux is multiplied by $E^{2.7}$ where $E$ is the kinetic energy per nucleon. Error bars of CALET data points represent the sum in quadrature of statistical and systematic uncertainties. The dashed blue lines represent the extrapolation of a SPL function fitted to data in the energy range $[25,200] \mathrm{GeV} / n . \Delta \gamma$ is the change of the spectral index above the transition energy $E_{0}$, represented by the vertical green dashed line. The error interval for $E_{0}$ from the DPL fit is shown by the green band.

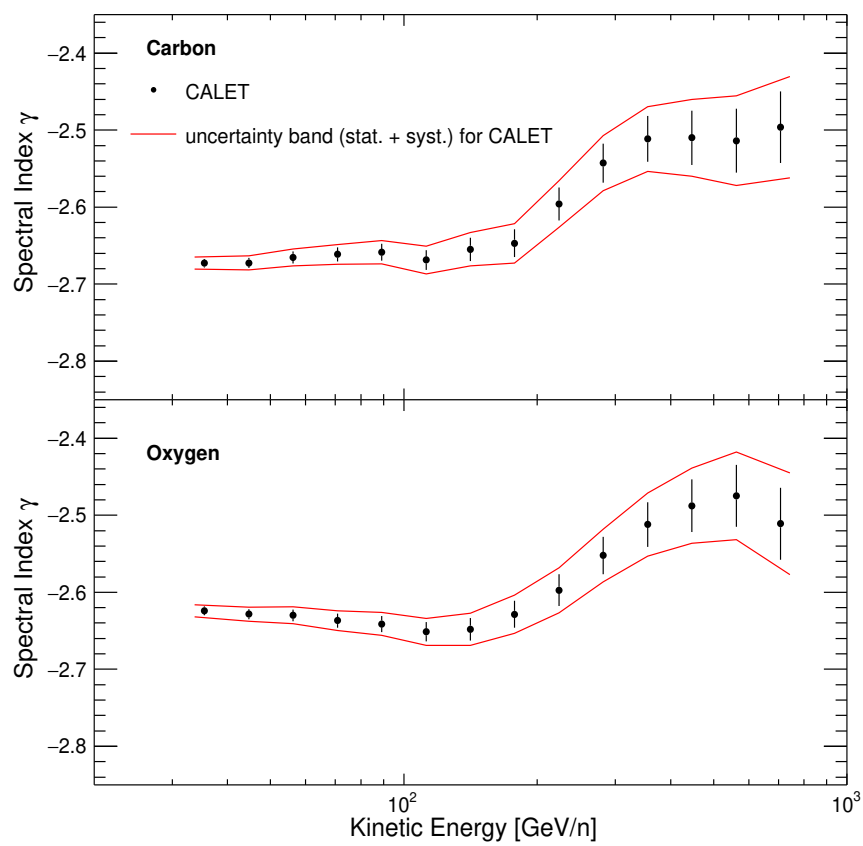

Figure 3: Energy dependence of the spectral index calculated within a sliding energy window for CALET (a) C and (b) O data. The spectral index is determined for each bin by fitting the data using \pm 3 bins. Red curves indicate the uncertainty range including systematic errors. 
it can stem from trigger inefficiencies, differences between MC simulation packages or hadronic models, or lacking modelling of the instrument.

\section{References}

[1] S. Torii and P. S. Marrocchesi (CALET), Adv. Space Res. 64, 2531 (2019).

[2] O. Adriani et al. (CALET), Phys. Rev. Lett. 119, 181101 (2017).

[3] O. Adriani et al. (CALET), Phys. Rev. Lett. 120, 261102 (2018).

[4] O. Adriani et al. (CALET), Phys. Rev. Lett. 122, 181102 (2019).

[5] P. Maestro and N. Mori (CALET), in Proceedings of Science (ICRC2017) 208 (2017).

[6] P. S. Marrocchesi et al., Nucl. Instr. and Meth. A 659, 477 (2011).

[7] Y. Akaike (CALET), in Proceedings of Science (ICRC2015) 613 (2015).

[8] O. Adriani et al. (CALET), Phys. Rev. Lett. 125, 251102 (2020).

[9] EPICS http://cosmos.n.kanagawa-u.ac.jp/EPICSHome/.

[10] S. Roesler, R. Engel, and J. Ranft, in Proceedings of the Monte Carlo Conference, Lisbon, 1033-1038 (2000).

[11] A. Ferrari, P. R. Sala, A. Fassò, and J. Ranft, Tech. Rep. CERN-2005-10, INFN/TC_05/11, SLAC-R-773 (2005).

[12] J. Allison et al., Nucl. Instr. and Meth. A 835, 186 (2016).

[13] G. D’Agostini, Nucl. Instr. and Meth. A 362, 487 (1995).

[14] M. Aguilar et al. (AMS), Phys. Rev. Lett. 119, 251101 (2017).

[15] J. J. Engelmann et al. (HEAO-3-C2), Astron. Astrophys. 233, 96 (1990).

[16] D. Müller et al., Astrophys. J. 374, 356 (1991).

[17] O. Adriani et al. (PAMELA), Astrophys. J. 93, 791 (2014).

[18] E. Atkin et al. (NUCLEON), JCAP 2017, 020 (2017).

[19] M. Simon et al., Astrophys. J. 239, 712 (1980).

[20] A. Panov et al. (ATIC), Bull. Russian Acad. Sci. 73, 564 (2009).

[21] M. Ave et al. (TRACER), Astrophys. J. 678, 262 (2008).

[22] H. S. Ahn et al. (CREAM), Astrophys. J. 707, 593 (2009). 


\section{Full Authors List: CALET}

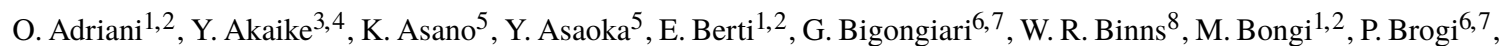
A. Bruno ${ }^{9,10}$, J. H. Buckley ${ }^{8}$, N. Cannady ${ }^{11,12,13}$, G. Castellini ${ }^{14}$, C. Checchia ${ }^{6}$, M. L. Cherry ${ }^{15}$, G. Collazuol ${ }^{16,17}$, K. Ebisawa ${ }^{18}$, A. W. Ficklin ${ }^{15}$, H. Fuke ${ }^{18}$, S. Gonzi ${ }^{1,2}$, T. G. Guzik ${ }^{15}$, T. Hams ${ }^{11}$, K. Hibino ${ }^{19}$, M. Ichimura ${ }^{20}$, K. Ioka ${ }^{21}$, W. Ishizaki ${ }^{5}$, M. H. Israel ${ }^{8}, K_{\text {K. Kasahara }}^{22}$, J. Kataoka ${ }^{23}$, R. Kataoka ${ }^{24}$, Y. Katayose ${ }^{25}$, C. Kato ${ }^{26}$, N. Kawanaka ${ }^{27,28}$ Y. Kawakubo ${ }^{15}$, K. Kobayashi ${ }^{3,4}$, K. Kohri ${ }^{29}$, H. S. Krawczynski ${ }^{8}$, J. F. Krizmanic ${ }^{11,12,13}$, J. Link ${ }^{11,12,13}$, P. Maestro ${ }^{6,7}$, P. S. Marrocchesi ${ }^{6,7}$, A. M. Messineo ${ }^{30,7}$, J.W. Mitchell ${ }^{12}$, S. Miyake ${ }^{32}$, A. A. Moiseev $33,12,13$, M. Mori ${ }^{34}$, N. Mori' ${ }^{2}$, H. M. Motz ${ }^{35}$, K. Munakata ${ }^{26}$, S. Nakahira ${ }^{18}$, J. Nishimura ${ }^{18}$, G. A. de Nolfo 9 , S. Okuno ${ }^{19}$, J. F. Ormes ${ }^{36}$, N. Ospina ${ }^{16,17}$, S. Ozawa ${ }^{37}$, L. Pacini ${ }^{1,14,2}$, P. Papini ${ }^{2}$, B. F. Rauch ${ }^{8}$, S. B. Ricciarini ${ }^{14,2}$, K. Sakai ${ }^{11,12,13}$, T. Sakamoto ${ }^{38}$, M. Sasaki ${ }^{33,12,13}$

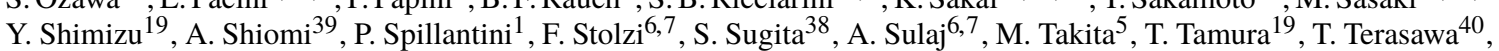
S. Torii ${ }^{3}$, Y. Tsunesada ${ }^{41}$, Y. Uchihori ${ }^{42}$, E. Vannuccini ${ }^{2}$, J. P. Wefel ${ }^{15}$, K. Yamaoka ${ }^{43}$, S. Yanagita ${ }^{44}$, A. Yoshida ${ }^{38}$, K. Yoshida ${ }^{22}$, and W. V. Zober ${ }^{8}$

${ }^{1}$ Department of Physics, University of Florence, Via Sansone, 1, 50019 Sesto, Fiorentino, Italy, ${ }^{2}$ INFN Sezione di Florence, Via Sansone, 1, 50019 Sesto, Fiorentino, Italy, ${ }^{3}$ Waseda Research Institute for Science and Engineering, Waseda University, 17 Kikuicho, Shinjuku, Tokyo 162-0044, Japan, ${ }^{4}$ JEM Utilization Center, Human Spaceflight Technology Directorate, Japan Aerospace Exploration Agency, 2-1-1 Sengen, Tsukuba, Ibaraki 305-8505, Japan, ${ }^{5}$ Institute for Cosmic Ray Research, The University of Tokyo, 5-1-5 Kashiwa-no-Ha, Kashiwa, Chiba 277-8582, Japan, ${ }^{6}$ Department of Physical Sciences, Earth and Environment, University of Siena, via Roma 56, 53100 Siena, Italy, ${ }^{7}$ INFN Sezione di Pisa, Polo Fibonacci, Largo B. Pontecorvo, 3, 56127 Pisa, Italy, ${ }^{8}$ Department of Physics and McDonnell Center for the Space Sciences, Washington University, One Brookings Drive, St. Louis, Missouri 63130-4899, USA, ${ }^{9}$ Heliospheric Physics Laboratory, NASA/GSFC, Greenbelt, Maryland 20771, USA, ${ }^{10}$ Department of Physics, Catholic University of America, Washington, DC 20064, USA, ${ }^{11}$ Center for Space Sciences and Technology, University of Maryland, Baltimore County, 1000 Hilltop Circle, Baltimore, Maryland 21250, USA, ${ }^{12}$ Astroparticle Physics Laboratory, NASA/GSFC, Greenbelt, Maryland 20771, USA, ${ }^{13}$ Center for Research and Exploration in Space Sciences and Technology, NASA/GSFC, Greenbelt, Maryland 20771, USA, ${ }^{14}$ Institute of Applied Physics (IFAC), National Research Council (CNR), Via Madonna del Piano, 10, 50019 Sesto, Fiorentino, Italy, ${ }^{15}$ Department of Physics and Astronomy, Louisiana State University, 202 Nicholson Hall, Baton Rouge, Louisiana 70803, USA, ${ }^{16}$ Department of Physics and Astronomy, University of Padova, Via Marzolo, 8, 35131 Padova, Italy, ${ }^{17}$ INFN Sezione di Padova, Via Marzolo, 8, 35131 Padova, Italy, ${ }^{18}$ Institute of Space and Astronautical Science, Japan Aerospace Exploration Agency, 3-1-1 Yoshinodai, Chuo, Sagamihara, Kanagawa 252-5210, Japan, ${ }^{19}$ Kanagawa University, 3-27-1 Rokkakubashi, Kanagawa, Yokohama, Kanagawa 221-8686, Japan, ${ }^{20}$ Faculty of Science and Technology, Graduate School of Science and Technology,, Hirosaki University, 3, Bunkyo, Hirosaki, Aomori 036-8561, Japan, ${ }^{21}$ Yukawa Institute for Theoretical Physics, Kyoto University, Kitashirakawa Oiwakecho, Sakyo, Kyoto 606-8502, Japan, ${ }^{22}$ Department of Electronic Information Systems, Shibaura Institute of Technology, 307 Fukasaku, Minuma, Saitama 337-8570, Japan, ${ }^{23}$ School of Advanced Science and Engineering, Waseda University, 3-4-1 Okubo, Shinjuku, Tokyo 169-8555, Japan, ${ }^{24}$ National Institute of Polar Research, 10-3, Midori-cho, Tachikawa, Tokyo 190-8518, Japan, ${ }^{25}$ Faculty of Engineering, Division of Intelligent Systems Engineering, Yokohama National University, 79-5 Tokiwadai, Hodogaya, Yokohama 240-8501, Japan, ${ }^{26}$ Faculty of Science, Shinshu University, 3-1-1 Asahi, Matsumoto, Nagano 390-8621, Japan, ${ }^{27}$ Hakubi Center, Kyoto University, Yoshida Honmachi, Sakyo-ku, Kyoto 606-8501, Japan, ${ }^{28}$ Department of Astronomy, Graduate School of Science, Kyoto University, Kitashirakawa Oiwake-cho, Sakyo-ku, Kyoto 6068502, Japan, ${ }^{29}$ Institute of Particle and Nuclear Studies, High Energy Accelerator Research Organization, 1-1 Oho, Tsukuba, Ibaraki 305-0801, Japan, ${ }^{30}$ University of Pisa, Polo Fibonacci, Largo B. Pontecorvo, 3, 56127 Pisa, Italy, ${ }^{31}$ Astroparticle Physics Laboratory, NASA/GSFC, Greenbelt, Maryland 20771, USA, ${ }^{32}$ Department of Electrical and Electronic Systems Engineering, National Institute of Technology, Ibaraki College, 866 Nakane, Hitachinaka, Ibaraki 312-8508, Japan ${ }^{33}$ Department of Astronomy, University of Maryland, College Park, Maryland 20742, USA, ${ }^{34}$ Department of Physical Sciences, College of Science and Engineering, Ritsumeikan University, Shiga 525-8577, Japan, ${ }^{35}$ Faculty of Science and Engineering, Global Center for Science and Engineering, Waseda University, 3-4-1 Okubo, Shinjuku, Tokyo 169-8555, Japan, ${ }^{36}$ Department of Physics and Astronomy, University of Denver, Physics Building, Room 211, 2112 East Wesley Avenue, Denver, Colorado 80208-6900, USA, ${ }^{37}$ Quantum ICT Advanced Development Center, National Institute of Information and Communications Technology, 4-2-1 Nukui-Kitamachi, Koganei, Tokyo 184-8795, Japan, ${ }^{38}$ College of Science and Engineering, Department of Physics and Mathematics, Aoyama Gakuin University, 5-10-1 Fuchinobe, Chuo, Sagamihara, Kanagawa 252-5258, Japan, ${ }^{39}$ College of Industrial Technology, Nihon University, 1-2-1 Izumi, Narashino, Chiba 275-8575, Japan ${ }^{40}$ RIKEN, 2-1 Hirosawa, Wako, Saitama 351-0198, Japan, ${ }^{41}$ Division of Mathematics and Physics, Graduate School of Science, Osaka City University, 3-3-138 Sugimoto, Sumiyoshi, Osaka 558-8585, Japan, ${ }^{42}$ National Institutes for Quantum and Radiation Science and Technology, 4-9-1 Anagawa, Inage, Chiba 263-8555, Japan, ${ }^{43}$ Nagoya University, Furo, Chikusa, Nagoya 464-8601, Japan, ${ }^{44}$ College of Science, Ibaraki University, 2-1-1 Bunkyo, Mito, Ibaraki 310-8512, Japan 